\title{
Multipliers of periodic orbits of quadratic polynomials and the parameter plane
}

\author{
Genadi Levin \\ Inst. of Math., Hebrew University, Jerusalem 91904, Israel
}

September 4, 2018

\begin{abstract}
We prove an extension result for the multiplier of an attracting periodic orbit of a quadratic map as a function of the parameter. This has applications to the problem of geometry of the Mandelbrot and Julia sets. In particular, we prove that the size of $p / q$-limb of a hyperbolic component of the Mandelbrot set of period $n$ is $O\left(4^{n} / p\right)$, and give an explicit condition on internal arguments under which the Julia set of corresponding (unique) infinitely renormalizable quadratic polynomial is not locally connected.
\end{abstract}

\section{Introduction}

Douady-Hubbard-Sullivan (DHS) theorem [3, [1] states that the multiplier $\rho$ of an attracting periodic orbit is a conformal isomorphism from a hyperbolic component of the Mandelbrot set onto the unit disk $\{|\rho|<1\}$, and it extends homeomorpically to the boundaries.

In Theorem 4 we prove that $\rho$ extends further to an analytic isomorphism from a region containing the hyperbolic component onto a simply connected domain $\tilde{\Omega}_{n}$ containing $\{|\rho| \leq 1\} \backslash\{1\}$, such that the domain $\tilde{\Omega}_{n}$ is explicitely defined by the period $n$ of the attracting orbit. This follows from the Main Inequality, see Theorem 3 below, which in turn is based upon Theorem 2 of [11, see formulation in Subsection 3.1 below. We derive from Theorem 4 few consequences including stated below Theorems 1 . 2 .

Let us be more precise. A hyperbolic component $W$ of period $n$ is a component of the interior of the Mandelbrot set $M$, such that, for $c$ in $W$, the quadratic map $f_{c}(z)=z^{2}+c$ has an attracting periodic orbit of period $n$. Denote by $\rho_{W}(c)$ 
the multiplier of this orbit of $f_{c}, c \in W$. By DHS theorem, $\rho_{W}$ performs a homeomorphism of the closure of $W$ onto the closed unit disk. A number $t$ is called an internal argument of a point $c \in \partial W$ iff $\rho_{W}(c)=\exp (2 \pi i t)$. The point $c$ with $t=0$ is called the root of $W$. If $c \in \partial W$ is not the root and has a rational internal argument $t$, the connected component of $M \backslash\{c\}$ which is disjoint with $W$ is not empty and called the $t$-limb $L(W, t)$ of $W$.

The first consequence of Theorem 4 concerns the size of the limbs. It strengthens Yoccoz's bound (off the root), see Section 5, particularly, part (b) of Proposition 2 and Comment 8 , .

Theorem 1 There exists $A>0$, such that, for every hyperbolic component $W$ of period $n$ and every $t=p / q \in[-1 / 2,1 / 2] \backslash\{0\}$, the diameter of the limb $L(W, t)$ is bounded by:

$$
\operatorname{diam} L(W, t) \leq A \frac{4^{n}}{p}
$$

This bound immediately implies the local connectivity of the Mandelbrot set at some parameters $c_{*}$, where $f_{c_{*}}$ is infinitely renormalizable with prescribed unbounded combinatorics, see Corollary 5.1 .

Let's discuss another result. Douady and Hubbard proposed an inductive construction to build an infinitely renormalizable quadratic map with non locally connected Julia set, and such that the Mandelbrot set is locally connected at this parameter [20], [16]. Their construction consists in choosing a sequence of internal arguments $t_{m}$ of successive bifurcations step by step, by continuity in the corresponding dynamical planes and in the parameter plane (with help of Yoccoz's bound). Next statement makes explicit the two sides of Douady-Hubbard's construction (parameter and dynamical).

Theorem 2 Let $n \geq 1$. Let

$$
t_{0}, t_{1}, \ldots, t_{m}, \ldots
$$

be any sequence of rational numbers $t_{m}=p_{m} / q_{m} \in(-1 / 2,1 / 2]$ which satisfy the following properties: if we denote $n_{0}=n, n_{m}=n q_{0} \ldots q_{m-1}, m>0$, then, for all $m$ large enough,

$$
p_{m}>\left(2 B_{0}\right) 4^{n_{m}}, \quad 2 n_{m}^{2}<\frac{p_{m}^{2}}{q_{m}},
$$

(where the constant $B_{0}$ is taken from Theorem 3 below), and also

$$
\sum_{m=1}^{\infty}\left|t_{m}\right|^{1 / q_{m-1}}<\infty .
$$

Given a hyperbolic component $W$ of the Mandelbrot set of period $n$, consider the following sequence of hyperbolic components $W^{m}: W_{0}=W$, and, for $m>0, W^{m}$ touches the hyperbolic component $W^{m-1}$ at the point with an internal argument 
$t_{m-1}$. For every $m$, consider the $t_{m}$-limb $L\left(W^{m}, t_{m}\right)$ of $W^{m}$ (it contains $\left.W^{m+1}\right)$. Then the limbs $L\left(W^{m}, t_{m}\right)$ shrink to a unique point $c_{*}$, the Mandelbrot set is locally connected at $c_{*}$, and the map $f_{c_{*}}$ is infinitely renormalizable with non locally connected Julia set.

This statement can be reformulated in term of the combinatorial data of a fixed infinitely renormalizable map, see Theorem 8

Let us comment on the inequalities ( 22)-( 3) above. Conditions ( 21) guarantee that the corresponding multipliers are local parameters. Namely, if $\rho_{W^{m}}(c)$ is the multiplier of the periodic orbit of $f_{c}$, which is attracting for $c \in W^{m}$, and $\psi_{m}$ denotes an inverse to $\log \rho_{W^{m}}$, then the first inequality in (2) ensures that $\psi_{m}$ extends as a holomorphic function to a disk of radius proportional to $n_{m} / q_{m}$ around $2 \pi i t_{m}$ while the second inequality in (2) implies that this analytic continuation is in fact injective. In turn, condition (3) will guarantee that the bifurcated periodic orbits stay away from the origin. It confirms Milnor's "guess" from [16], p. 21.

\section{$1.1 \quad$ Notations}

We collect some notations to be used throughout the paper.

$f_{c}(z)=z^{2}+c, J_{c}=J\left(f_{c}\right)$ the Julia set, $D_{\infty}(c)$ the basin of infinity, $M=\left\{c \in \mathbf{C}: J_{c}\right.$ connected $\}$ is the Mandelbrot set,

$B_{c}$ is the Bottcher coordinate at infinity normalized by $B_{c}(z) \sim z$ as $z \rightarrow \infty$, $G_{c}=\lim _{n \rightarrow \infty} 2^{-n} \log \left|f_{c}^{n}(z)\right|$ Green's function of $D_{\infty}(c), G(z) \sim \log |z|$ at $\infty$ extended by 0 to the whole plane, so that $G_{c}(z)=\log B_{c}(z)$ near infinity.

$B(a, r)=\{z:|z-a|<r\}$.

\section{Main inequality}

Let $O=\left\{b_{k}\right\}_{k=1}^{n}$ be a periodic orbit of $f=f_{c_{0}}$ of exact period $n$. Its multiplier is the number $\rho=\left(f^{n}\right)^{\prime}\left(b_{k}\right)$. If $\rho \neq 1$, by the Implicit function theorem, there exist $n$ holomorphic functions $b_{k}(c), k=1, . ., n$ defined in a neighborhood of $c_{0}$, such that $O(c)=\left\{b_{k}(c)\right\}_{k=1}^{n}$ is a periodic orbit of $f_{c}$, and $O\left(c_{0}\right)=O$. In particular, if $\rho(c)=\left(f_{c}^{n}\right)^{\prime}\left(b_{k}(c)\right)=2^{n} b_{1}(c) \ldots b_{n}(c)$ denotes the multiplier of $O(c)$, it is holomorphic in $c$ in this neighborhood.

In what follows we assume that the multiplier $\rho$ is not very big because we are interested in studying the behavior of a multiplier not far from the hyperbolic component where the corresponding periodic orbit is attracting. So, we will always assume that

$$
|\rho|<e
$$


Theorem 3 There exist $\lambda_{*}, B, B_{0}$ as follows. Let $O(c)$ be a repelling periodic orbit of $f_{c}$ of exact period $n$, and the multiplier of $O(c)$ is equal to $\rho$. Then the following inequality holds:

$\left|n \rho-n+2 c \frac{\rho^{\prime}(c)}{\rho(c)}\right| \leq K_{n}(c)\left\{\log |\rho(c)|+\frac{\left|\rho^{\prime}(c)\right|}{|\rho(c)|} \frac{1}{\pi} \operatorname{area}\left(\left\{z: 0<G_{c}(z)<2^{-n} \log \lambda_{*}\right\}\right)\right\}$

Here

$$
K_{n}(c)=\frac{2}{\log \lambda_{*}} \max \left\{\left|\left(f_{c}^{n}\right)^{\prime}(z)\right|: G_{c}\left(f_{c}^{n}(z)\right)=2 \log \lambda_{*}\right\},
$$

and we have:

$$
K_{n}(c) \leq B(2 \beta)^{n}
$$

where $\beta$ is the unique positive solution of the equation $\beta^{2}-|c|=\beta$, and also

$$
K_{n}(c) \leq B_{0} 4^{n}
$$

Comment 1 Let us notice for the future use few bounds related to the number $G_{c}(0)$.

(a) First, the following fact was established in [6]: for the multiplier $\rho$ of every repelling periodic orbit of $f_{c}$ of period $n$, we have

$$
G_{c}(0) \leq \frac{1}{n} \log |\rho|
$$

In particular, since we assume $|\rho|<e$, then $c$ belongs to a neighborhood of $M$ where $G_{c}(0) \leq n^{-1} \leq 1$.

(b) Second, the transfinite diameter of the set $\left\{z: 0 \leq G_{c}(z)<2^{-n} \log \lambda\right\}$ $(\lambda>1)$ is equal to $\lambda^{2^{-n}}$, and, hence, by a theorem of Polya [8],

$$
\operatorname{area}\left(\left\{z: 0 \leq G_{c}(z)<2^{-n} \log \lambda\right\}\right) \leq \pi \lambda^{2^{-n+1}} .
$$

Consider two particular cases of the inequality ( 4). One case corresponds to passing to a limit as $|\rho| \rightarrow 1$, and in another one put $\rho^{\prime}=0$. Then we get the following corollary.

Corollary 2.1 (A) There is $C_{0}$, such that, if, for some $c_{0},\left|\rho\left(c_{0}\right)\right|=1$, then

$$
\left|\rho^{\prime}\left(c_{0}\right)\right| \geq C_{0} \frac{n\left|\rho\left(c_{0}\right)-1\right|}{K_{n}\left(c_{0}\right)}
$$

(B) For every $n$-periodic orbit $O$ of $f_{c}$ with the multiplier $\rho$, if $1<|\rho|<e$ and

$$
n|\rho-1|>K_{n}(c) \log |\rho|,
$$

then $\rho^{\prime} \neq 0$.

Comment 2 (A) implies, of course, that $\rho^{\prime} \neq 0$ on the boundary of a hyperbolic component where $|\rho|=1$, and it was known after [4]. 


\section{Proof of Theorem 3}

\subsection{Derivative of multiplier and Ruelle operator}

The proof is based on the following result, see Theorem 2 of [11]. Consider the Ruelle transfer operator

$$
T g(z)=\sum_{w: f_{c}(w)=z} \frac{g(w)}{\left(f_{c}^{\prime}(w)\right)^{2}}
$$

Given a periodic orbit $O=\left\{b_{k}\right\}_{k=1}^{n}$ of $f$ of exact period $n$ and with the multiplier $\rho \neq 0,1$, associate to $O$ a function

$$
A(z)=A_{O}(z)=\sum_{k=1}^{n} \frac{1}{\left(z-b_{k}\right)^{2}}+\frac{1}{\rho(1-\rho)} \sum_{k=1}^{n} \frac{\left(f^{n}\right)^{\prime \prime}\left(b_{k}\right)}{z-b_{k}}
$$

where $\left(f^{n}\right)^{\prime \prime}(z)$ is the second derivative of the $n$-iterate of $f$ w.r.t. $z$. Then

$$
A(z)=(T A)(z)+2 \frac{\rho^{\prime}}{\rho}(T 1)(z) .
$$

Comment 3 In [11] we use this to give another, local, proof of DHS theorem.

\subsection{Two identities}

Denote

$$
\gamma_{k}=\frac{\left(f^{n}\right)^{\prime \prime}\left(b_{k}\right)}{\rho(1-\rho)} .
$$

Let's find the coefficients $C_{1}$ and $C_{2}$ in the expansion of the function $A$ at infinity:

$$
A(z)=C_{1} z^{-1}+C_{2} z^{-2}+O\left(|z|^{-3}\right) .
$$

Since

$$
A(z)=\sum_{k=1}^{n}\left(\frac{1}{\left(z-b_{k}\right)^{2}}+\frac{\gamma_{k}}{z-b_{k}}\right)
$$

then

$$
C_{1}=\sum_{k=1}^{n} \gamma_{k}, \quad C_{2}=n+\sum_{k=1}^{n} b_{k} \gamma_{k}
$$

On the other hand, comparing the expansions at $\infty$ in (9), one can see that

$$
C_{1}=\frac{\rho^{\prime}}{\rho}, \quad C_{2}=2 c \frac{\rho^{\prime}}{\rho} .
$$

Thus we have

$$
\sum_{k=1}^{n} \gamma_{k},=\frac{\rho^{\prime}}{\rho}, \quad \sum_{k=1}^{n} b_{k} \gamma_{k}=-n+2 c \frac{\rho^{\prime}}{\rho}
$$




\subsection{Estimate from above}

Fix $c$ and denote $f=f_{c}, G=G_{c}$ etc. Given $\lambda>1$, denote $U_{\lambda}=\{z: 0<G(z)<$ $\log \lambda\}$ and $C_{\lambda}=U_{\lambda} \backslash f^{-1}\left(U_{\lambda}\right)$. Introduce also a number associated to the cycle $O$ as follows:

$$
M=\liminf _{\delta \rightarrow 0} \int_{B(b, \delta) \backslash f_{O}^{-n}(B(b, \delta))} \frac{d \sigma_{z}}{|z-b|^{2}}
$$

where $b$ is a point of the orbit, and $d \sigma_{z}$ is the area element in the $z$-plane, $b$ is any point in $O$, and $f_{O}^{-n}$ is a branch defined near $b$ that fixes $b$. It is easy to see that

$$
M \leq 2 \pi \log |\rho|
$$

Comment 4 One can show (though we'll not use this fact below) that $M=$ $2 \pi \alpha \log |\rho|$ where $\alpha$ is the the density of $D_{\infty}$ at the point $b$ in the logarithmic metric [13].

Lemma 3.1 For every $\lambda>1$,

$$
\int_{C_{\lambda}}|A(z)| d \sigma_{z} \leq M+2 \frac{\left|\rho^{\prime}\right|}{|\rho|} \operatorname{area}\left(\left\{z: 0<G(z)<\frac{1}{2} \log \lambda\right\}\right)
$$

Proof. Denote by $f_{O}^{-i}$ a local branch sending a point $b$ of the orbit ) to $f^{n-i}(b)$, $i=1,2, \ldots, n$. For $\delta>0$, let $V_{\delta}=U_{\lambda} \backslash \cup_{i=0}^{n-1} f_{O}^{-i}(B(b, \delta))$. Then $A$ is integrable in $V_{\delta}$, and one can write

$$
\int_{V_{\delta}}|A| d \sigma=\int_{V_{\delta}}\left|T A(z)+2 \frac{\rho^{\prime}}{\rho} T 1(z)\right| d \sigma \leq \int_{f^{-1}\left(V_{\delta}\right)}|A| d \sigma+2 \frac{\left|\rho^{\prime}\right|}{|\rho|} \operatorname{area}\left(f^{-1}\left(V_{\delta}\right)\right) .
$$

Note that $V_{\delta}=f^{-1}\left(V_{\delta}\right) \cup C_{\lambda} \cup \Delta \backslash\left(B(b, \delta) \backslash f_{O}^{-n}(B(b, \delta))\right.$ where $\Delta$ is an open set, which shrinks to $-O=\left(-b_{1}, \ldots,-b_{n}\right)$ as $\delta \rightarrow 0$. Therefore,

$$
\int_{C_{\lambda}}|A| d \sigma \leq \liminf _{\delta \rightarrow 0} \int_{B(b, \delta) \backslash f_{O}^{-n}(B(b, \delta))}|A(z)| d \sigma_{z}+2 \frac{\left|\rho^{\prime}\right|}{|\rho|} \operatorname{area}\left(U_{\lambda^{1 / 2}}\right) .
$$

We have: $A(z)=(z-b)^{-2}+r(z)$ where $r(z)$ is integrable at $b$. Sending $\delta \rightarrow 0$, we get the desired inequality.

\subsection{Estimate from below}

We are left with the problem to estimate the integral

$$
I(\lambda):=\int_{C_{\lambda}}|A(z)| d \sigma_{z}
$$


from below.

To be able to deal with $I(\lambda)$ when $J$ is disconnected and $\lambda$ close to 1 , let us extend the Bottcher function $B$ of $f$ at $\infty$ to the following simply connected domain (on the Riemann sphere) $D_{\infty}^{*}$ : it is obtained from $D_{\infty}$ by deleting all arcs of external rays (gradient curves of $G$ ) starting from 0 and all its preimages up to the Julia set. Denote the extended Bottcher function again by $B$. It maps $D_{\infty}^{*}$ onto a domain $A$ whose boundary is a 'hedgehog', see [14], [15]. Let $\phi: A \rightarrow D_{\infty}^{*}$ be the inverse map. Note that $\phi^{\prime}$ has a singularity at the vertex $p$ of every needle (i.e. the image by $B$ of a critical point of $G_{c}$ ), but it is of the type $|w-p|^{-1 / 2}$, i.e. integrable against the area.

Let $\lambda>1$. One writes:

$$
I(\lambda)=\int_{C_{\lambda}}|A(z)| d \sigma_{z}=\int_{\lambda<|w|<\lambda^{2}}|A(\phi(w))|\left|\phi^{\prime}(w)\right|^{2} d \sigma_{w}
$$

Let us assume that $f^{n}(\phi(w)) \neq 0$ for every $\lambda \leq|w| \leq \lambda^{2}$, in other words, that $\lambda^{2^{n}}>G(0)$. By Fubini theorem, we can continue as follows:

$$
\int_{\lambda<|w|<\lambda^{2}}|A(\phi(w))|\left|\phi^{\prime}(w)\right|^{2} d \sigma_{w} \geq \int_{\lambda}^{\lambda^{2}} \min _{|w|=r} \frac{\left|\phi^{\prime}(w)\right|}{\left|f^{n}(\phi(w))\right|} d r \int_{\{z: G(z)=\log r\}}\left|A(z) f^{n}(z)\right||d z| .
$$

Now,

$$
\int_{\{z: G(z)=\log r\}}\left|A(z) f^{n}(z)\right||d z| \geq\left|\int_{\{z: G(z)=\log r\}} A(z) f^{n}(z) d z\right|=2 \pi|R|,
$$

where

$$
R=\frac{1}{2 \pi i} \int_{\{z: G(z)=\log r\}} A(z) f^{n}(z) d z .
$$

By the definition of $A$ and the Cauchy formula,

$$
R=\sum_{k=1}^{n}\left(\left(f^{n}\right)^{\prime}\left(b_{k}\right)+\gamma_{k} f^{n}\left(b_{k}\right)\right)=n \rho-n+2 c \frac{\rho^{\prime}}{\rho}
$$

where we use the identity (10). Thus, for every $\lambda>1$, such that $\lambda^{2^{n}}>G(0)$,

$$
I(\lambda) \geq 2 \pi|R| \int_{\lambda}^{\lambda^{2}} \min _{|w|=r} \frac{\left|\phi^{\prime}(w)\right|}{\left|f^{n}(\phi(w))\right|} d r .
$$

Now, from $f \phi(w)=\phi\left(w^{2}\right)$ we conclude that

$$
\frac{\phi^{\prime}(w)}{f^{n}(\phi(w))}=\frac{2^{n} w^{2^{n}-1} \phi^{\prime}\left(w^{2^{n}}\right)}{\phi\left(w^{2^{n}}\right)\left(f^{n}\right)^{\prime}(\phi(w))} .
$$

There is a choice for $\lambda>1$. Take $\lambda=\lambda_{n}$ where $\lambda_{n}^{2^{n}}=\lambda_{*}$ and $\lambda_{*}$ is fixed. 
Let us estimate $|c|$. By Comment 1, (a), $G_{c}(0) \leq n^{-1}$. If $J_{c}$ is connected, then $|c| \leq 2$. Otherwise the function $\Psi_{c}$ which is inverse to the Bottcher coordinate $B_{c}$ of $f_{c}$ extends in a univalent fashion to the disk $\left\{|w|>\exp \left(G_{c}(0)\right)\right\}$. Besides, $c=\Psi\left(w_{c}\right)$, for some $\left|w_{c}\right|=\exp \left(2 G_{c}(0)\right)$, and $\Psi$ is odd. Therefore, by a classical distortion theorem, see e.g. [8],

$$
|c|=\left|\Psi\left(w_{c}\right)\right| \leq 2 \exp \left(2 G_{c}(0)\right) \leq 2 \exp \left(\frac{2}{n}\right), n>0 .
$$

Thus $c$ belongs to a bounded neighborhood of the Mandelbrot set. Hence, we can fix $\lambda_{*}$ in such a way that for all $c$ in the fixed neighborhood, $\left|\phi^{\prime}(w) / \phi(w)\right|>$ $1 /(2|w|)$ for all $\lambda_{*}<|w|<\lambda_{*}^{2}$. We define

$$
K_{n}(c)=\frac{2 \max \left\{\left|\left(f_{c}^{n}\right)^{\prime}(z)\right|: G_{c}(z)=2^{-n+1} \log \lambda_{*}\right\}}{\log \lambda_{*}} .
$$

The inequality (4) follows.

\subsection{The constant}

It remains to estimate $K_{n}(c)$. Let us show that there is $B$ such that

$$
K_{n}(c) \leq B(2 \beta)^{n}
$$

where $\beta$ is a unique positive solution of the equation $\beta^{2}-|c|=\beta$. (Note that $\beta>1$ because $c \neq 0$.) Indeed, if, for some $\delta>0,|z|=\beta+\delta$, then $\left|f_{c}(z)\right|>\beta+2 \beta \delta$. Hence, if $\left|f_{c}^{n}(z)\right|=R$ where $R>1+\beta$, then it follows $\left|f_{c}^{k}(z)\right|-\beta<(R-\beta)(2 \beta)^{k-n}$. Let us choose and fix $R$, such that $R>1+\beta$ and $\left\{z: G_{c}(z)=2 \log \lambda_{*}\right\} \subset B(0, R)$ for every $c$ from the neighborhood of $M$. Then we get (16) with

$$
B=\frac{2}{\log \lambda_{*}} \Pi_{k=1}^{\infty}\left(1+\frac{R}{2^{k}}\right)
$$

On the other hand, as we know,

$$
|c| \leq 2 \exp \left(\frac{2}{n}\right) \leq 2\left(1+\frac{A_{1}}{n}\right)
$$

for some $A_{1}$. Therefore,

$$
\beta \leq 2+\frac{A_{2}}{n}
$$

and, hence,

$$
K_{n}(c) \leq B 4^{n}\left(1+\frac{A_{2}}{2 n}\right)^{n} \leq 4^{n} B_{0},
$$

for some $A_{2}$ and $B_{0}$. 


\section{Analytic extension of the multiplier}

\subsection{A set of domains}

Given $C>1$, consider an open set of points $\Omega$ in the punctured $\rho$-plane defined by the inequality

$$
|\rho-1|>C \log |\rho|
$$

It obviously contains the set $D_{*}=\{\rho: 0<|\rho| \leq 1, \rho \neq 1\}$ and is disjoint with an interval $1<\rho<1+\epsilon$. Denote by $\Omega_{C}$ a connected component of $\Omega$ which contains the set $D_{*}$ completed by 0 . Denote also by $\Omega_{C}^{\log }$ the set of points

$$
L=\log \rho=x+i y, \quad \rho \in \Omega_{C}, \quad|y| \leq \pi
$$

Note that $\Omega_{C}^{\log }$ is symmetric w.r.t. the real axis.

Lemma 4.1 $\Omega_{C}$ is simply-connected. More precisely, the intersection of $\Omega_{C}^{\log }$ with any vertical line with $x=x_{0}>0$ is either empty or equal to two (mirror symmetric) intervals. If $C>2$, then $x<2 /(C-2)$ for all $L=x+$ iy in $\Omega_{C}^{\log }$. If $C$ is large enough, $\Omega_{C}^{\log }$ contains two (mirror symmetric) domains bounded by the lines $y= \pm(C / 2) x(x>0)$ and $y= \pm \pi$

Proof. In the $\log$-coordinate $L=\log \rho=x+i y,|y| \leq \pi$, the condition (17) with $x>0$ is equivalent to

$$
\sin ^{2}\left(\frac{y}{2}\right)>\frac{C^{2} x^{2}-(\exp (x)-1)^{2}}{4 \exp (x)}
$$

Given $x$, the set of $y$ which satisfies the latter inequality is either empty or a union $[-\pi,-y) \cup(y, \pi]$ with some $y>0$. We have for $x+i y \in \Omega_{C}^{\log }: 1 \geq\left(C^{2} x^{2}-\right.$ $\left.(\exp (x)-1)^{2}\right) / 4 \exp (x)$, and since $x>0$, then $C x<\exp (x)+1$. If $C>2$, it implies $x<2 /(C-2)$. If $C$ large, then by this $x>0$ must be small, and the boundary curve of $\Omega_{C}^{\log }$ can be written in the form $\sin (y / 2)= \pm(1 / 2)\left(C^{2}-1\right)^{1 / 2} x(1-x / 2+x r(x, C))$ where $r(x, C)=o\left(1 / C^{2}\right)$ uniformly in $x$. The statement follows.

\subsection{Hyperbolic components and related definitions}

Let $W$ be a component of the interior of $M$. It is called a hyperbolic component if $f_{c}, c \in W$, has an attracting periodic orbit $O(c)$. Let us call $W n$-hyperbolic, if the exact period of the latter orbit is $n$.

Denote by $\rho_{W}(c)$ the multiplier of $O(c)$. By the DHS theorem, $\rho_{W}$ is a analytic isomorphism of $W$ onto the unit disk, and it extends homeomorphically to the 
boundaries. Given a number $t \in(-1 / 2,1 / 2]$, denote by $c(W, t)$ the unique point in $\partial W$ with the internal argument $t$, i.e. $\rho_{W}$ at this points is equal to $\exp (2 \pi t)$. Root of $W$ is the point $c_{W}=c(W, 0)$ with the internal argument zero. $W$ is called primitive iff its root is not a point of other hyperbolic component.

If $t=p / q$ is a rational number, we will always assume that $p, q$ are co-primes. For any rational $t \neq 0$, denote by $L(W, t)$ the connected component of $M \backslash\{c(W, t)\}$ which is disjoint with $W$. It is called the $t$-limb of $W$. Denote also by $W(t)$ a $n q$ hyperbolic component with the root point $c(W, t)$; it touches $W$ at this point. The limb $L(W, t)$ contains $W(t)$. The root $c_{W}$ of $W$ is the landing point of precisely two external rays of the Mandelbrot set [4]. In what follows, it will be important the notion of the wake of a hyperbolic component $W$ [10]: it is the only component $W^{*}$ of the plane cut by two external rays to the root of $W$ that contains $W$. We'll use also the following fact (see e.g. [15], Theorem 7.2):

Proposition 1 The points of periodic orbit $O(c)$ as well as its multiplier $\rho_{W}$ extend as analytic functions to the wake $W^{*}$. Moreover, $\left|\rho_{W}\right|>1$ in $W^{*} \backslash \bar{W}$.

The map $\rho_{W}$ from $W$ onto the unit disk $c \mapsto \rho_{W}(c)$ has an inverse, which we denote by $c=\psi_{W}(\rho)$. It is defined so far in the unit disk.

\subsection{Extension of the multiplier}

Introduce

$$
\Omega_{n}=\Omega_{n^{-1} 4^{n} B_{0}}=\left\{\rho:|\rho-1|>\frac{4^{n} B_{0}}{n} \log |\rho|\right\}
$$

and

$$
\Omega_{n}^{\log }=\Omega_{n^{-1} 4^{n} B_{0}}^{\log }=\left\{L=x+i y: \exp (L) \in \Omega_{n},|y| \leq \pi\right\} .
$$

Theorem 4 (a) The function $\psi=\psi_{W}$ extends to a holomorphic function in the domain $\Omega_{n}$.

(b) The function $\psi$ is univalent in a subset $\tilde{\Omega}_{n}$ of $\Omega_{n}$ defined by its log-projection $\tilde{\Omega}_{n}^{\log }=\left\{\log \rho: \rho \in \tilde{\Omega}_{n}\right\}$ as follows:

$$
\tilde{\Omega}_{n}^{\log }=\Omega_{n}^{\log } \backslash\left\{L:\left|L-R_{n}\right|<R_{n}\right\}
$$

where $R_{n}$ depends on $n$ only and has an asymptotics

$$
R_{n}=\left(2+O\left(2^{-n}\right)\right) n \log 2
$$

as $n \rightarrow \infty$.

Finally, the image of $\tilde{\Omega}_{n}$ by $\psi$ is contained in the wake $W^{*}$. 
Comment 5 The disk $\left\{L:\left|L-R_{n}\right|<R_{n}\right\}$ cuts off from $\Omega_{n}^{\log }$ an asymptotically negligible portion: the deleted part is contained in the disk $\left\{|L|<r_{n}\right\}$, where $r_{n} \sim\left(4 \log 2 / B_{0}\right)\left(n^{2} / 4^{n}\right)$.

Proof. Let $\rho$ be a multiplier of some repelling orbit of period $n$ for some $f_{c}$. By Corollary 2.1, part (B), if $\rho \in \Omega_{n}$, then $\rho^{\prime} \neq 0$.

(a) Since $\Omega_{n}$ is simply connected, it is enough to show that $\psi$ has an analytic extension along any curve in $\Omega_{n}$ (which starts at 0 ).

Firstly, $c$ is an algebraic function of $\rho$. Indeed, consider two functions $Q(c, b)=$ $f_{c}^{n}(b)-b$ and $P(c, b, \rho)=b f_{c}(b) \ldots f_{c}^{n-1}(b)-\rho / 2^{n}$. They are polynomials in $c, b$ and $\rho$, which are of degrees $2^{n}$ and $2^{n}-1$ respectively w.r.t. $b$ and with leading coefficients 1 . Hence, resultant $R(c, \rho)$ of $Q$ and $P$ w.r.t. $b$ is a polynomial such that $R(c, \rho)=0$ if and only if $Q$ and $P$ have a joint root $b$, which means that $b$ is a fixed point of $f_{c}^{n}$ and $\rho=\left(f_{c}^{n}\right)^{\prime}(b)$.

Now assume that $\psi$ does not have an analytic continuation along a (simple) curve $\beta$ in $\Omega_{n}$ starting in 0 . By the above, it means that $\beta$ contains a singular point $\rho_{0}$ of the algebraic function $c$, that is, when we make a small loop around $\rho_{0}$ then we get a different value of $c$. Let $\rho_{0}$ be the first such point when we move from 0 . Denote $c_{0}=\psi\left(\rho_{0}\right)$ the limit value of $\psi$ when $\rho$ approaches $\rho_{0}$ along $\beta$. Since $c$ is algebraic, $c$ and $\rho$ can be written in a form:

$$
c-c_{0}=u^{j}, \rho-\rho_{0}=(\phi(u))^{k}
$$

where the integers $j, k>0$ and $\phi$ is holomorphic near $u=0$ such that $\phi(0)=$ $0, \phi^{\prime}(0) \neq 0$. Note that $\rho_{0} \neq 1$, hence $\rho$ is holomorphic in $c$ in a neighborhood of $c_{0}$. By the same reason, the period of the corresponding periodic orbit of $f_{c_{0}}$ (=limit of the orbit of $f_{\psi(\rho)}$ when $\rho$ tends to $\rho_{0}$ along $\beta$ ) is exactly $n . \rho \in \Omega_{n}$, hence, $\rho^{\prime}\left(c_{0}\right) \neq 0$. But $d c / d \rho \sim(j / k) u^{j-k} / \phi^{\prime}(0)$, which implies $j=k$. Since $\rho$ is holomorphic in $c$ near $c_{0}$, we get

$$
(\phi(u))^{k}=\tilde{\phi}\left(u^{k}\right)
$$

for another holomorphic near $u=0$ function $\tilde{\phi}$. Here $\tilde{\phi}^{\prime}(0) \neq 0$ because $\phi^{\prime}(0) \neq 0$. Thus in a new local coordinate $\tilde{u}=u^{k}$ we have $c-c_{0}=\tilde{u}, \rho-\rho_{0}=\tilde{\phi}(\tilde{u})$. It follows $\psi$ can be extended through $\rho_{0}$, a contradiction.

(b) $\psi$ is univalent in some domain if it takes values in a simply connected domain in the $c$-plane where the function $\rho(c)$ (local inverse to $\psi$ ) is well-defined. Let us choose as such a domain in the $c$-plane the wake of $W$. The choice is correct because as we know the function $\rho$ extends to a holomorphic function from $W$ to its wake $W^{*}$. Now it is enough to show that, for any $L=\log \rho \in \tilde{\Omega}_{n}^{\log }$, the value $\psi(\rho)$ cannot belong to the boundary of $W^{*}$. The latter consists of two external rays in the $c$-plane to the root of $W$ (including the root itself). Assume it is not the case. Then we find a curve $l$ in $\tilde{\Omega_{n}}$, which starts at 0 and ends at some $\rho_{0}$, 
such that $\psi(\rho) \in W^{*}$ for all $\rho \in l \backslash\left\{\rho_{0}\right\}$ while $c_{0}=\psi\left(\rho_{0}\right)$, for $L_{0}=\log \rho_{0} \in \Omega_{n}^{\log }$ does belong to such a ray. Consider a continuation of the $n$-periodic orbit $O(c)$ of $f_{c}$ along the curve $l$ which is attracting for $c \in W$. Then the rotation number of the periodic orbit $O\left(c_{0}\right)$ of $f_{c_{0}}$ is zero (see e.g. Remark 7.2 of [15]). Now, by Theorem 5.1 of [14,

$$
\frac{\left|L_{0}\right|^{2}}{R e\left(L_{0}\right)} \leq \frac{2 \pi n \log 2}{\pi / 2-\arctan \left[\left(2^{n}-1\right) a_{0} / \pi\right]}
$$

where $a_{0}=G_{c_{0}}(0)$. Let's estimate $a_{0}$ from above. According to [6], Theorem 1.6, $\operatorname{Re}\left(L_{0}\right)=\log \left|\rho_{0}\right| \geq n a_{0}$. By Lemma 4.1, $\operatorname{Re}\left(L_{0}\right)<2 /\left(C_{n}-2\right)$, where $C_{n}=4^{n} B_{0} / n$. Hence, $a_{0}<2 /\left(4^{n} B_{0}-2 n\right)$. It allows us to define

$$
R_{n}=\frac{\pi n \log 2}{\pi / 2-\arctan \left[\frac{2\left(2^{n}-1\right)}{\pi\left(4^{n} B_{0}-2 n\right)}\right]} .
$$

Note that $R_{n}=\left(2+O\left(2^{-n}\right)\right) n \log 2$ as $n \rightarrow \infty$. Now, ([19) implies that $\left|L_{0}-R_{n}\right|<$ $R_{n}$, i.e. $\rho_{0}$ belongs to the part of $\Omega_{n}$ that we delete, a contradiction.

\section{Limbs}

\subsection{Yoccoz's circles}

Let $W$ be an $n$-hyperbolic component. As we know, the multiplier $\rho_{W}$ is defined and analytic throughout the wake $W^{*}$. Let us formulate a result due to Yoccoz, which is a basic point for further considerations. For every $t=p / q \neq 0$, consider the limb $L(W, t)$. Then, for every $c \in L(W, t)$, a branch of $\log \rho_{W}(c)$ is contained in the following round disk (Yoccoz's circle):

$$
Y_{n}(t)=\left\{L:\left|L-\left(2 \pi i t+\frac{n \log 2}{q}\right)\right|<\frac{n \log 2}{q}\right\}
$$

see [10], [18] as well as [19, [1], 7], 6], 15].

As a well known corollary [10, we have:

Proposition 2 (a) The intersection of the wake $W^{*}$ of $W$ (completed by the root) with $M$ is equal to the union of $\bar{W}$ and its limbs.

(b) For every hyperbolic component $W$ there exists a constant $C_{W}$ depending on $W$, such that, if $W$ is not primitive, then

$$
\operatorname{diam} L(W, p / q) \leq \frac{C_{W}}{q}
$$

and if $W$ is primitive, then

$$
\operatorname{diam} L(W, p / q) \leq \frac{C_{W}}{q^{1 / 2}}
$$




\subsection{Condition on the internal argument}

Definition 5.1 Given $n$, let us call a rational numbers $t=p / q \in[-1 / 2,1 / 2]$ $n$-deep, iff, for every $n^{\prime} \leq n$ and every $n^{\prime}$-hyperbolic component $W$, there is a disk $B(2 \pi i t, d)$ with $d<\pi$, such that the following holds:

(a) the image $\exp (B(2 \pi i t, d / 2))$ of $B(2 \pi i t, d / 2)$ by the exponential map covers the limb $L(W, t)$,

(b) the inverse $\psi_{W}$ to the map $\rho_{W}$ extends to a univalent function defined in the union of the unit disk and the domain $\exp (B(2 \pi i t, d))$.

Comment 6 By (20),

$$
d<\frac{4 n \log 2}{q}
$$

Conjecturely, $d$ should be comparable to $n / q^{2}$. For simplicity, we will always assume that

$$
d>\frac{n}{q^{4}}
$$

Comment 7 By the proof of Theorem 4, if $\psi_{W}$ extends just holomorphically into $\exp (B(2 \pi i t, d))$ and maps it into the wake $W^{*}$, then $t$ is $n$-deep. In particular, if $B(2 \pi i t,(4 n \log 2) / q) \subset \tilde{\Omega}_{n}^{\log }$, then $t$ is $n$-deep.

Proposition 3 (1) For every fixed n,

(1a) $n$-deep rationals are dense in $(-1 / 2,1 / 2)$;

(1b) the set of all $t$, which are not $n$-deep has a unique concentration point 0 .

(2) there exists $n_{0}$, such that, for every $n>n_{0}$, the point $t=p / q \in(-1 / 2,1 / 2)$ is n-deep if the following two inequalities hold:

$$
\begin{gathered}
p>4^{n}\left(2 B_{0}\right), \\
\frac{p^{2}}{q}>2 n^{2} .
\end{gathered}
$$

Proof. (1) follows from Comment 7. As for (2), by the same Comment it is enough to check that the inequalities ( 21) $-($ 22) guarantee that the disk of radius $(4 n \log 2) / q$ centered at the point $2 \pi i t$ is contained in the domain $\tilde{\Omega}_{n}^{\log }$. Denote $\theta=2 \pi t$ and consider the disk $B(i \theta, r)$. Then for $C>0$ and $L=i \theta+w$ where $|w|<r, r<1$, one can write:

$|\exp (L)-1|-C R e(L) \geq|\exp (-i \theta)-1|-|\exp (w)-1|-C|w| \geq 2|\sin (\theta / 2)|-(C+2) r$.

That is, if $r<2|\sin (\theta / 2)| /(C+2)$, then the disk $B(i \theta, r)$ lies inside of $\Omega_{C}^{\log }$. Note that $|\sin (\theta)| \geq 2 \theta / \pi$. This shows that if we put here $C=C_{n}=4^{n} B_{0} / n$ and $r=r_{n}=(4 n \log 2) / q$, then the inequality ( 21) ensures for $n$ big that $B\left(i \theta, r_{n}\right)$ lies in $\Omega_{n}^{\log }$. 
It is also easy to check that the inequality (22) means that $B\left(i \theta, r_{n}\right)$ is disjoint with the disk $B\left(R_{n}, R_{n}\right)$, for $n$ big, so that both inequalities imply that $B\left(i \theta, r_{n}\right)$ is contained in $\tilde{\Omega}_{n}^{\log }$.

\subsection{Uniform bound on the size of some limbs}

Theorem 5 (1) Let $W$ be an n-hyperbolic component, and let $c \in \partial W$ have an internal argument $t$, such that $t$ is $n$-deep. Then there is a topological disk $B(c)$, such that:

$B(c)$ does not contain the root $c_{W}$ of $W$ and it is "roughly" a round disk around the point c: for some $r$,

$$
B(c, r / 4) \subset B(c) \subset B(c, 4 r) .
$$

The function $\log \rho_{W}$ is univalent in $B(c)$ and maps it onto a disk $B(2 \pi i t, d / 2)$. Moreover, $\log \rho_{W}$ extends univalently to a topological disk containing $B(c)$, and maps this bigger domain onto $B(2 \pi i t, d)$.

The limb $L(W, t)$ is contained in $B(c)$.

(2) There exists $A>0$, such that, for every n-hyperbolic component $W$ and every $t=p / q \in[-1 / 2,1 / 2]$, the diameter of the limb $L(W, t)$ is bounded by:

$$
\operatorname{diam} L(W, t) \leq A \frac{4^{n}}{p}=A \frac{4^{n}}{t} \frac{1}{q}
$$

Comment 8 Part (2) is Theorem 1 announced in the Introduction. It strengthens the bound of part (b) of Proposition 2 (off the root, i.e. if $t=p / q$ is outside of a neighborhood of zero).

Proof. (1) Following the Definition 5.1, one can take $B(c)=\psi_{W}(\exp (B(2 \pi i t, d / 2)))$. We use here and later on the following classical distortion bounds for univalent maps, see e.g. [8]: if $g$ is univalent in a disk $B(0, R)$ and $r<R$, then

$$
B\left(g(0), \alpha(r / R)^{-1} r\left|g^{\prime}(0)\right|\right) \subset g(B(0, R)) \subset B\left(g(0), \alpha(r / R) r\left|g^{\prime}(0)\right|\right)
$$

where $\alpha(x)=(1-x)^{-2}$.

Now (1) is an immediate consequence of the definition 5.1 and the latter bound (with $r=R / 2$ ).

(2) Let us prove the second part of the Theorem. It is enough to show that there exist $M, N$, such that if $p>M 4^{n}$, then $\operatorname{diam} L(W, t) \leq N 4^{n} / p$ for all $n, t$. Fix $n$ and $t=p / q$. In the course of the proof $A_{i}$ will denote different constants from a finite collection of numbers. For $r_{n}=2 n \log 2 / q$ and $C_{n}=n^{-1} 4^{n} B_{0}$ one can write:

$$
\frac{r_{n}\left(C_{n}+2\right)}{|\sin (\pi p / q)|} \leq A_{1} \frac{4^{n}}{p}
$$


Hence, if $A_{1} 4^{n} / p<1 / 4$, then by ( 23) the disk $B\left(2 \pi i p / q, 2 r_{n}\right)$ is contained in $\Omega_{n}^{\log }$. Hence, so is the disk $Y:=\{|L-(i \theta+n \log 2 / q)|<n \log 2 / q\}$ which is contained in $B\left(2 \pi i p / q, 2 r_{n}\right)$. By Theorem $4, \psi$ extends to a holomorphic function in $B\left(2 \pi i p / q, 2 r_{n}\right)$. For every $c$ in the limb $L(W, t)$ there is $L \in Y$ such that $c=\psi(L)$. Therefore, the distance between the root of the limb and $c$ is bounded from above by the integral $J:=\int_{1 \theta}^{L}\left|\psi^{\prime}(w)\right||d w|$ for some $L$ with $|L|=r_{n}$. On the other hand, by Theorem 3 ,

$$
\left|\psi^{\prime}(w)\right| \leq \frac{\left|2 c n^{-1}\right|+\pi^{-1} \operatorname{area}\left(\left\{z: G_{c}(z)<a 2^{-n}\right\}\right) C_{n}}{|\exp (w)-1|-C_{n} \operatorname{Re}(w)}
$$

Here area $\operatorname{area}\left(\left\{z: G_{c}(z)<a 2^{-n}\right\}\right)$ is smaller than $\pi(1+o(1))$ as $n \rightarrow \infty$ by Comment 1 (b). Using (23) we can write

$$
J \leq(1+o(1)) C_{n} r_{n} \int_{0}^{1} \frac{d \tau}{2|\sin (\theta / 2)|-r_{n}\left(C_{n}+2\right) \tau}
$$

and hence

$$
J \leq(1+o(1)) C_{n} \log \frac{2|\sin (\theta / 2)|}{2|\sin (\theta / 2)|-r_{n}\left(C_{n}+2\right)} .
$$

Since $r_{n}\left(C_{n}+2\right) /|\sin (\theta / 2)|<1 / 4$ and $\log (1-x)^{-1} \leq 2 x$ if $0<x<1 / 4$, we can proceed:

$$
J \leq(1+o(1)) C_{n} \frac{r_{n}}{|\sin (\theta / 2)|} \leq A_{2} \frac{4^{n}}{p}
$$

for all $n$. Thus it is enough to put $M=4 A_{1}$ and $N=A_{2}$.

We have the following corollary.

Corollary 5.1 For any sequence

$$
t_{0}, t_{1}, \ldots, t_{m}, \ldots
$$

of rational numbers $t_{m}=p_{m} / q_{m} \in[-1 / 2,1 / 2]$ the following holds. Let $W$ by a n-hyperbolic component. Let $W^{0}=W, W^{m}=W^{m-1}\left(t_{m-1}\right), m=1,2, \ldots$ (i.e. the hyperbolic component $W^{m}$ touches the hyperbolic component $W^{m-1}$ at a point with the internal argument $\left.t_{m-1}\right)$, so that $W^{m}$ is $n_{m}$-hyperbolic component where the periods $n_{0}=n, n_{m}=n q_{0} \ldots q_{m-1}$. Assume that

$$
\lim _{m \rightarrow \infty} \frac{p_{m}}{4^{n_{m}}}=\infty
$$

Then the limbs $L\left(W^{m}, t_{m}\right)$ shrink to a unique point $c_{*}$. The Mandelbrot set is locally connected at $c_{*}$.

Proof. By the previous result, $\operatorname{diam} L\left(W^{m}, t_{m}\right) \rightarrow 0$. Hence, $L\left(W^{m}, t_{m}\right)$ shrink to a point $c_{*}$. The local connectivity follows because these limbs form a shrinking sequence of connected neighborhoods of $c_{*}$. 


\section{$6 \quad$ Selecting internal arguments}

\subsection{Bifurcations}

Throughout this subsection, we consider the following situation. Let $W$ be a $n$ hyperbolic component, and let $c_{0} \in \partial W$ have an internal argument $t_{0}=p / q \neq 0$.

Let $O(c)=\left\{b_{j}(c)\right\}_{j=1}^{n}$ be the periodic orbit of $f_{c}$ which is attracting when $c \in W$. Then all $b_{j}(c)$ as well as the mulptiplier $\rho(c)$ are holomorphic in $W$ and extend to holomorphic functions in $c$ near $c_{0}$ (in fact, in the whole wake of $W$ ). As we know the multiplier $\rho(c)$ is injective near $c_{0}$. Denote the inverse function by $\psi(\rho), \psi\left(\exp \left(2 \pi t_{0}\right)\right)=c_{0} . \psi$ is well defined in $\tilde{\Omega}_{n}$, that includes the unit disk and a neighborhood of the point $\rho_{0}=\exp \left(2 \pi i t_{0}\right)$.

Consider now the wake of the $n q$-hyperbolic component $W\left(t_{0}\right)$ (by definition, it tangents $W$ at the point $\left.c_{0}\right)$. By Douady-Hubbard theory [4, the root $c_{0}$ of $W\left(t_{0}\right)$ is the landing point of precisely two external rays of $M$. Denote their arguments by $\tau\left(c_{0}\right), \tilde{\tau}\left(c_{0}\right)$. For every $c$ in the wake of $W\left(t_{0}\right)$, we have the following picture in the dynamical plane of $f_{c}$ : two external rays of $f_{c}$ with the arguments $\tau\left(c_{0}\right)$ and $\tilde{\tau}\left(c_{0}\right)$ land at one point (denote it $b_{n}(c)$ ), which is a point of the repelling periodic orbit $O(c)$. It implies also that $c$ lies in a component of the plane minus these two external rays which does not contain the origin. Indeed, by the formula for the uniformization map of the exterior of $M$ [3], this is true for those $c$ in the wake of $W\left(t_{0}\right)$ which are outside of the Mandelbrot set. Hence, it must be true throughout the wake because $c$ cannot cross the external rays as well as their landing point $b_{n}(c)$.

We will make use also of a well known formula, see e.g. [15]:

$$
\left|\tau\left(c_{0}\right)-\tilde{\tau}\left(c_{0}\right)\right|=\frac{(\beta-\alpha)\left(2^{n}-1\right)}{2^{n q}-1}
$$

where $\beta, \alpha \in\left\{0,1,2, \ldots, 2^{n}-1\right\}$ are two "digits" determined by $W$.

Next statement is combinatorial (cf. [2]).

Lemma 6.1 Let $c$ be a point of a limb $L\left(W, t^{\prime}\right)$ with some $t^{\prime}=p^{\prime} / q^{\prime}$ and $q^{\prime}>2$. Assume that $f_{c}$ has a periodic point of period $n Q$ with the multiplier 1 . Then

$$
Q \geq q^{\prime}-1
$$

Proof. Consider the dynamical plane of $f_{c}$. The critical value $c$ belongs to a petal of $a$. Since $c$ lies in the sector bounded by the two external rays with arguments $\tau\left(c^{\prime}\right), \tilde{\tau}\left(c^{\prime}\right)$ where $c^{\prime}$ is the root of $L\left(W, t^{\prime}\right)$, then $a$ is in the same sector, too. On the other hand, $a$ is a landing point of two external rays fixed by $f_{n}^{n Q}$. Therefore, there must be $\left|\tau\left(c^{\prime}\right)-\tilde{\tau}\left(c^{\prime}\right)\right|>\left(2^{n Q}-1\right)^{-1}$. If we apply now the formula (27), it gives $n Q \geq n q^{\prime}-2 n+1$, that is, $Q \geq q^{\prime}-1$. 
Next Lemma describes the bifurcation near the parameter $c_{0}$, cf. [2], [9].

Lemma 6.2 There exist a small disk $U$ around the origin, a neighborhood $V$ of the cycle $O\left(c_{0}\right)$, and $n$ functions $F_{k}(s), k=1, \ldots, n$, which are holomorphic in $U$, such that $F_{k}(0)=0, F_{k}^{\prime}(0) \neq 0$ and, for every $s \in U$ and every $\rho=\rho_{0}+s^{q}$, the points

$$
b_{k}\left(c_{0}\right)+F_{k}(s \exp (2 \pi j / q)), k=1, \ldots, n, j=0, \ldots, q-1
$$

are the only fixed points of $f_{c}^{n q}$ in the neighborhood $V$ different from $O(c)$, where $c=\psi\left(\rho_{0}+s^{q}\right)$ and $\rho$ is the multiplier of $O(c)$. They form a periodic orbit of $f_{c}$ of period $n q$.

Proof. Introduce

$$
p(c, z)=\frac{f_{c}^{n q}(z)-z}{f_{c}^{n}(z)-z} .
$$

It is a polynomial in $z$ and $c$. As we also know, the function $c=\psi(\rho)$ satisfies another polynomial equation $R(c, \rho)=0$, see the proof of Theorem 4 . Hence, periodic points of period $n$ form an algebraic function in $\rho$ : they satisfy a polynomial equation of the form

$$
\tilde{p}(\rho, z)=0 .
$$

For every $k$, the point $\left(\rho_{0}, b_{k}\left(c_{0}\right)\right)$ with $\rho_{0}=\exp \left(2 \pi i t_{0}\right)$ is a singular point of $\tilde{p}$. Hence, there exist a local uniformizing parameter $s$ and co-prime $i, j$, such that every solution of $(28)$ in a small enough neighborhood of the singular point has a form

$$
\rho-\rho_{0}=s^{i}, z-b_{k}\left(c_{0}\right)=F_{k}(s)
$$

where

$$
F_{k}(t)=r_{k} s^{j}+O\left(s^{j+1}\right)
$$

is a holomorphic function near $0, r_{k} \neq 0$. Let us show that necessarily $i=q, j=1$.

Introduce a new (local) variable $w=L(z)=z-b_{k}(c)$ and consider conjugate map

$$
g(\rho, w)=L \circ f_{c}^{n} \circ L^{-1}(w)
$$

where $c=\psi(\rho)$. Then for all $\rho$ near $\rho_{0}$ and $w$ near 0 ,

$$
g(\rho, 0)=0, \frac{\partial g}{\partial w}(\rho, 0)=\rho .
$$

Now consider $g^{q}$. Then

$$
g^{q}\left(\rho_{0}, w\right)=w+A w^{q+1}+O\left(w^{q+2}\right)
$$

where $A \neq 0$. Taking this into account we obtain using (29) that

$$
g^{q}(\rho, w)-w=\left(\rho^{q}-1\right) w+O\left(\left(\rho-\rho_{0}\right) w^{2}\right)+A w^{q+1}+O\left(w^{q+2}\right) \quad A \neq 0 .
$$


On the other hand, $b_{k}(c)-b_{k}\left(c_{0}\right)=O\left(\rho-\rho_{0}\right)=O\left(s^{i}\right)$ so that a pair of functions

$$
\left(\rho-\rho_{0}=s^{i}, w=r_{k} s^{j}+O\left(s^{j+1}\right)+O\left(s^{i}\right)\right)
$$

is a solution of the equation $\left(g^{q}(\rho, w)-w\right) / w=0$. Substituting this pair of functions of $s$ into ( 30 ) we arriv at the conclusion that $i=j q$.

Thus locally (near $\left.\left(\rho_{0}, b_{k}\left(c_{0}\right)\right)\right)$ the algebraic function $(\rho, z)$ has the form

$$
\rho-\rho_{0}=s^{q}, z-b_{k}\left(c_{0}\right)=F_{k}(s)=F_{k}^{\prime}(0) s+O\left(s^{2}\right), F_{k}^{\prime}(0) \neq 0
$$

Now, $n q$ points $b_{k}\left(c_{0}\right)+F_{k}(s \exp (2 \pi j / q)), k=1, \ldots, n, j=0, \ldots, q-1$ are fixed by $f_{c}^{n q}$ and are close to the $n$-cycle of $f_{c_{0}}$ with a multiplier a primitive $q$-root of 1 . Therefore, they form a single $n q$-periodic orbit.

The following two Lemmas allow us to connect the multiplier $\rho$ with the multiplier of a periodic orbit after the bifurcation. In the first one we use an idea of [2] although our proof is more involved than in [2]. The reason is that the limb $L\left(W, t_{0}\right)$ may contain more than one hyperbolic components of period $n q$ or less (which is impossible in case $n=1$ considered in [2]).

Lemma 6.3 There exists $n_{0}$ as follows. For every $n>n_{0}$ and $q>n_{0}$, if $t_{0}=p / q$ is $n$-deep, then each function $F_{k}$ extends to a holomorphic function in the disk $\{|s|<9 / 10\}$. Moreover, the domain $\psi\left(B\left(\rho_{0},(9 / 10)^{q}\right)\right)$ is disjoint with any limb $L\left(W, p^{\prime} / q^{\prime}\right)$ which is different from $L\left(W, t_{0}\right)$ and such that $q^{\prime} \leq q+1$.

Proof. We prove by several steps that the function $F_{k}(s)$ extends to a analytic function in the disk $B\left(0, r^{1 / q}\right)$ where $r=1 /\left(n q^{2}(q+1)\right)$. It will be enough because $(9 / 10)^{q}<r$ for big $q>n$

Denote $B=B\left(\rho_{0}, r\right)$ and $\tilde{B}=\psi(B)$.

1. $\tilde{B}$ is disjoint with all the limbs $L\left(W, t^{\prime}\right)$ of $W$ different from $L\left(W, t_{0}\right)$, such that $t^{\prime}=p^{\prime} / q^{\prime}$ and $q^{\prime} \leq q+1$. Indeed, let us project the disk $B$ by log. Since $r$ is very small, the projection is (asymptotically) a disk $B\left(2 \pi i t_{0}, r\right)$. Doing a simple calculation similar to [2] we see that for all $n, q$, the latter disk is disjoint with all Yoccoz's circle that touches the vertical line at a point $2 \pi i p^{\prime} / q^{\prime}$ with some $q^{\prime} \leq q+1$. The claim follows.

2. Assume the contrary: there exists a path $\gamma$ in $B\left(0, r^{1 / q}\right)$ that connect 0 ans some $s_{1}$, such that $F_{k}$ cannot be extended analytically through $s_{1}$. Since $(\rho(s), a(s))$ where $\rho(s)-\rho_{0}=s^{q}, z(s)-b_{k}\left(c_{0}\right)=F_{k}(s)$ satisfy a polynomial equation, the function $F_{k}$ has an analytic continuation along every curve unless it meets a singular point. Therefore, the point $\left(\rho_{1}, a\right)$, such that $\rho_{1}:=\rho\left(s_{1}\right)$ and $a:=b_{k}\left(c_{0}\right)+F_{k}\left(s_{1}\right)$ is so that $a$ is a fixed point of $f_{c_{1}}^{n q}\left(c_{1}=\psi\left(\rho\left(s_{1}\right)\right)\right)$ with the multiplier 1. 
Let us see where $c_{1} \in B$ can be situated. By Lemma 6.1, $c_{1}$ belongs either to $L\left(W, t_{0}\right)$ or to some $L\left(W, p^{\prime} / q^{\prime}\right)$ other than $L\left(W, t_{0}\right)$ and such that $q^{\prime} \leq q+1$. The latter possibility is excluded by Step 1 . Thus $c_{1} \in L\left(W, t_{0}\right)$.

3. Consider the image $\Gamma$ of the path $\gamma$ by the map $\psi\left(s^{q}\right)$. It connects the root $c_{0}=c\left(W, t_{0}\right)$ of $L\left(W, t_{0}\right)$ to $c_{1}$. The curve $\Gamma$ cannot belong completely to the wake $W^{* *}:=W\left(t_{0}\right)^{*}$ (which contains the limb $L\left(W, t_{0}\right)$ ). The reason is that, as we know, the point $a(s)$ extends analytically to this wake. Assume for a moment that there is another curve $\Gamma_{1}$ that connect $c_{1}$ and $c_{0}$ inside the wake $W^{* *}$ and such that it lies in $\tilde{B}$. Let us deform (kepping the end points fixed) the curve $\Gamma_{1}$ to $\Gamma$ inside $\tilde{B}$. If along the way we will not meet another singular point, then it contradics to the fact that the point $c_{1}$ is singular for $a(s)$. Hence, we must meet another singulat point. It also must belong to the limb $L\left(W, t_{0}\right)$. Then we can replave $\Gamma_{1}$ by another curve that connects $c_{0}$ and $c_{1}$ inside $W^{* *}$ and inside $B$, such that $\Gamma_{1}$ can be deformed to $\Gamma$ inside $B$ without meeting singular points, a contradiction.

We conclude that there exists a curve $\Gamma_{1}$ connecting $c_{0}$ and $c_{1}$ inside the wake $W^{* *}$, such that it leaves $\tilde{B}$ (and then comes back to $c_{1}$ ), such that when we deform $\Gamma_{1}$ to $\Gamma$ we meet a singular point $c_{2}$, which belongs to another $\operatorname{limb} L\left(W, p_{2} / q_{2}\right)$ with $q_{2} \leq q+1$. Then the continuum $L\left(W, p_{2} / q_{2}\right)$ contains the points $c_{2}$ and its root and disjoint with $L\left(W, t_{0}\right)$, therefore, $L\left(W, p_{2} / q_{2}\right)$ must cross $\tilde{B}$, a contradiction to the fact that $\tilde{B}$ is disjoint with all such limbs.

Definition 6.1 The latter Lemma shows that for every $\rho$ such that $\rho-\rho_{0}=s^{q}$ with $|s|<9 / 10$, the points $b_{k}\left(c_{0}\right)+F_{k}(s \exp (2 \pi j / q)), k=1, \ldots, n, j=0, \ldots, q-1$ form a nq-cycle of $f_{c}$ where $c=\psi\left(\rho_{0}+s^{q}\right)$. We denote this periodic orbit by $O^{q}(c)$. As $c=c_{0}$, it coincides with the $n$-cycle $O\left(c_{0}\right)$.

Definition 6.2 If $A, B$ are two sets in the plane, we say that $A$ is $\delta$-close to $B$ and denote it by $d(A, B)<\delta$ iff for every point $a \in A$ there exists a point $b \in B$, such that $|a-b|<\delta$.

Comment 9 The function $d$ is not symmetric. On the other hand, it is easy to check the triangle inequality: if $d(A, B)<\delta_{1}$ and $d(C, A)<\delta_{2}$, then $d(C, B)<$ $\delta_{1}+\delta_{2}$.

For $t_{0}=p / q$, denote

$$
B_{t_{0}}=B\left(\exp \left(2 \pi i t_{0}\right),\left(\frac{9}{10}\right)^{q}\right)
$$

By ( 15), one can assume that $|c|<3$. If $z$ is a periodic point of $f_{c}$ we then have that $|z| \leq 6$. Now the Schwarz Lemma gives us: 
Corollary 6.1 For every $\rho \in B_{t_{0}}$, where $t_{0}$ is $n$-deep, and for every s such that $\rho-\rho_{0}=s^{q}$ and $|s|<9 / 10$, we have, for $c=\psi(\rho)$, we have:

$$
\begin{gathered}
d\left(O^{q}(c), O\left(c_{0}\right)\right)<7|s| \\
d\left(O(c), O\left(c_{0}\right)\right)<6\left(\frac{10|s|}{9}\right)^{q}
\end{gathered}
$$

Denote by $\lambda(\rho)$ the multiplier of the periodic orbit $O^{q}(c)$ for $c=\psi(\rho)$. In other words, $\lambda=\rho_{W\left(t_{0}\right)} \circ \rho^{-1}$ whenever it is well defined.

It follows from, for instance, Lemma 6.2 that $\lambda$ is defined and holomorphic near $\rho=\rho_{0}$. Moreover, by [9],

$$
\frac{d \lambda}{d \rho}\left(\rho_{0}\right)=-\frac{q^{2}}{\rho_{0}}
$$

This formula can be also derived directly from ( 30) with help of ( 31) where $i=q, j=1$.

As it follows directly from Lemma 6.3, for $n, q$ biger than $n_{0}$, the function $\lambda$ is holomorphic in $B_{t_{0}}$. But it is not necessarily univalent there. On the other hand, to choose next internal argument, we need that the image by $\lambda$ is not small. This is proved in the next statement.

Lemma 6.4 For every real $T \in(-1 / 2,1 / 2]$, the equation $\lambda(\rho)=\exp (2 \pi i T)$ has at most one solution $\rho$ in $B_{t_{0}}$, and for such a solution the corresponding $c=\psi(\rho)$ lies on the boundary of the nq-hyperbolic component $W\left(t_{0}\right)$, which tangents $W$ at the point $c_{0}=\psi\left(2 \pi i t_{0}\right)$. The following covering property holds: for every $r \leq(9 / 10)^{q}$, the image of the disk $B\left(\exp \left(2 \pi i t_{0}\right), r\right)$ under the map $\lambda$ covers the disk $B\left(1, q^{2} r / 16\right)$.

Proof. Let $\lambda\left(\rho_{1}\right)=\exp \left(2 \pi i T_{1}\right)$ for some real $T_{1}$. Lemma 6.3 tells us that $\psi\left(B_{t_{0}}\right)$ is disjoint with any $p^{\prime} / q^{\prime}$-limb of $W$ other than $L\left(W, t_{0}\right)$, where $q^{\prime} \leq q+1$. On the other hand, $f_{c_{1}}$ has $n q$-periodic orbit $O^{q}\left(c_{1}\right)$ with the multiplier $\rho_{1}=\exp \left(2 \pi i T_{1}\right)$. Therefore, $c_{1} \in L(W, t)$ and moreover lies in the boundary of an $n q$-hyperbolic component. This hyperbolic component belongs to some limb $L\left(W, t^{\prime}\right), t^{\prime}=p^{\prime} / q^{\prime}$, and $q^{\prime} \geq q+1$. If $t^{\prime} \neq t_{0}$, then $L\left(W, t^{\prime}\right)$ contains a parameter $\tilde{c}$ on the boundary of this component, such that $f_{\tilde{c}}$ has a $n q$-periodic orbit with the multiplier 1 . By Lemma 6.1. $q \geq q^{\prime}-1$, i.e. $q \geq q^{\prime}-1$, a contradiction with the previous inequality $q^{\prime} \geq q+1$. Thus $c_{1}$ is in the $\operatorname{limb} L\left(W, t_{0}\right)$. Hence, it lies in the boundary of some $n q$-hyperbolic component belonging this time to $L\left(W, t_{0}\right)$. On the other hand, the multiplier of the periodic orbit $O^{q}(c)$ is bigger on modulus than 1 off the closure of the component $W\left(t_{0}\right)$. Thus the hyperbolic component containing $c_{1}$ in its boundary is just $W\left(t_{0}\right)$. Since the corresponding multiplier is injective on the boundary, it means that $\rho_{1}$ is the only solution of the equation $\lambda(\rho)=\exp \left(2 \pi i T_{1}\right)$. 
To prove the covering property, given $r \leq(9 / 10)^{q}$, consider a function $m(w)=$ $\left.\left(q^{2} r\right)^{-1}\left(\lambda\left(2 \pi i t_{0}\right)+r w\right)-1\right)$. It is holomorphic in the unit disk, $m(0)=0$, $\left|m^{\prime}(0)\right|=1$ and, by the proved part of the statement, $m(w)=0$ if and only if $w=0$. Therefore, by a classical result (Caratheodory-Fekete, see e.g. [8]), the disk $B(0,1 / 16)$ is covered by the image of the unit disk under the map $m$. This is equivalent to the covering property.

\subsection{Non locally connected Julia sets}

Our aim is to prove Theorem 2 stated in the Introduction. We'll do it in few steps. The main one consists in proving the following result.

Theorem 6 There exists $N$, such that, for every $n>N$ the following holds. Let $t_{0}, t_{1}, \ldots, t_{m}, \ldots$ be any sequence of rational numbers $t_{m}=p_{m} / q_{m} \in(-1 / 2,1 / 2)$ satisfying the following property:

(a) if one denotes $n_{0}=n, n_{m}=n q_{0} q_{1} \ldots q_{m-1}$ for $m>0$, then $t_{m}$ is $n_{m}$-deep, $m=0,1,2, \ldots$, see Definition 5.1. Moreover, $\left|t_{m}\right|^{1 / q_{m-1}}<9 / 10, m=1,2, \ldots$;

Then, for every n-hyperbolic components $W$ :

(1) denote $W^{0}=W, W^{m}=W^{m-1}\left(t_{m-1}\right), m=1,2, \ldots$ (i.e. the hyperbolic component $W^{m}$ touches the hyperbolic component $W^{m-1}$ at a point with the internal argument $\left.t_{m-1}\right)$. Then (a) implies that the limbs $L\left(W^{m-1}, t_{m-1}\right)$ form a nested sequence of compact sets with the intersection a unique point $c_{*}$.

If in addition to (a) we have also:

(b)

$$
\sum_{m=1}^{\infty}\left|t_{m}\right|^{1 / q_{m-1}}<4^{-n} / 16
$$

then:

(2) the map $f_{c^{*}}$ is infinitely renormalizable with non locally connected Julia set.

We begin the proof with some notations.

$$
\begin{gathered}
\Delta=4^{-n} / 16, \quad \epsilon_{m}=\left|t_{m+1}\right|^{1 / q_{m}}, m=0,1, \ldots, \quad n_{0}=n, n_{m}=n q_{0} \ldots q_{m-1}, m=1,2, \ldots \\
\psi_{m}(w)=\psi_{W^{m}}(\exp (w)), m=0,1, \ldots \\
c_{m}=\psi_{m}\left(2 \pi i t_{m}\right), m=0,1, \ldots
\end{gathered}
$$

$c_{m}$ is the point in the boundary of $W^{m}$ with the internal argument $t_{m}$. 
Since $t_{m}$ is $n_{m}$-deep, the function $\psi_{m}$ extends in a univalent fashion to $B\left(2 \pi i t_{m}, d_{m}\right)$. Remind that $n_{m} / q_{m}^{4}<d_{m}<4 n_{m} \log 2 / q_{m}$ and

$$
B\left(c_{m}\right)=\psi_{m}\left(B\left(2 \pi i t_{m}, d_{m} / 2\right)\right) .
$$

By Theorem 5, each $B\left(c_{m}\right)$ is "roughly" a round disk around the point $c_{m}$ :

$$
B\left(c_{m}, r_{m} / 4\right) \subset B_{m} \subset B\left(c_{m}, 4 r_{m}\right)
$$

where $r_{m}=\left|\psi_{m}^{\prime}\left(2 \pi i t_{m}\right)\right| d_{m} / 2$.

$$
B_{m}=\psi_{m}\left(B\left(2 \pi i t_{m},(9 / 10)^{q_{m}}\right)\right), \quad D_{m}=\psi_{m}\left(B\left(2 \pi i t_{m},\left|t_{m+1}\right|\right)\right), \quad D_{m}^{\prime}=\psi_{m}\left(B\left(2 \pi i t_{m}, 100\left|t_{m+1}\right|\right)\right)
$$

Note that if $r / d_{m}<1 / 2$, then $\psi_{m}\left(B\left(\left(2 \pi i t_{m}, r\right)\right)\right.$ is "almost" a disk:

$$
B\left(c_{m}, 4^{-1} r / r_{m}\right) \subset \psi_{m}\left(B\left(\left(2 \pi i t_{m}, r\right)\right) \subset B\left(c_{m}, 4 r / r_{m}\right)\right.
$$

Note also that by the condition (a) all $q_{m}$ are big provided $n$ is big.

It implies $100\left|t_{m+1}\right| /(9 / 10)^{q_{m}}$ and $(9 / 10)^{q_{m}} / d_{m}$ are small (for $n$ big enough), $\tilde{B}_{m}, D_{m}$ and $D_{m}^{\prime}$ are "almost" disks (around $c_{m}$ ), and for $m=0,1, \ldots$,

$$
D_{m} \subset D_{m}^{\prime} \subset B_{m} \subset B\left(c_{m}\right),
$$

and, moreover, the diameter of each previous set is much smaller than the diameter of the next one.

Lemma 6.5 There is $N$, such that, for each $n>N$ the following holds.

(i) $c_{m+1} \in D_{m}, m=0,1 \ldots$

(ii) $B\left(c_{m+1}\right) \subset D_{m}^{\prime}, m=0,1, \ldots$

Proof. We use Lemma 6.4. Consider the function $\lambda=\rho_{W^{m+1}} \circ \psi_{m}$. Then $\lambda$ is holomorphic in $B\left(2 \pi i t_{m},(9 / 10)^{q_{m}}\right)$ and since $\left|t_{m+1}\right|=\epsilon_{m}^{q_{m}}<(9 / 10)^{q_{m}}$, then $\lambda\left(B\left(2 \pi i t_{m},\left|t_{m+1}\right|\right)\right)$ covers the disk $B\left(1, q_{m}^{2}\left|t_{m+1}\right| / 16\right)$. If $q_{m}>16$ and $t_{m+1}$ is small enough, then

$$
\exp \left(2 \pi i t_{m+1}\right) \sim 1+2 \pi i t_{m+1} \in B\left(1, q_{m}^{2}\left|t_{m+1}\right| / 16\right) .
$$

Therefore, the point

$$
c_{m+1}=\psi_{m+1}\left(2 \pi i t_{m+1}\right) \in \psi_{m}\left(B\left(2 \pi i t_{m},\left|t_{m+1}\right|\right)\right)=D_{m}
$$

To prove that $B\left(c_{m+1}\right)$ is contained in the 100-times bigger disk $D_{m}^{\prime}$, let us notice that by part (1) of Theorem [5, the root $c_{m}$ of $W^{m+1}$ is outside of 
$B\left(c_{m+1}\right)$, and $B\left(c_{m+1}\right)$ is "roughly" a disk around $c_{m+1}$. Hence, $B\left(c_{m+1}\right) \subset$ $B\left(c_{m+1}, 4\left|c_{m+1}-c_{m}\right|\right)$ and $B\left(c_{m+1}\right) \subset B\left(c_{m}, 17\left|c_{m+1}-c_{m}\right|\right)$

Let us estimate $\left|c_{m+1}-c_{m}\right|$. We use the distortion bounds for univalent maps (25). Denote $\delta_{m}=2\left|t_{m+1}\right| / d_{m}$. Since $c_{m+1} \in D_{m}$, then $\left|c_{m+1}-c_{m}\right|<\delta_{m} \alpha\left(\delta_{m}\right) r_{m}$ where $\alpha(x)=(1-x)^{-2}$. Thus $B\left(c_{m+1}\right) \subset B\left(c_{m}, 17 \delta_{m} \alpha\left(\delta_{m}\right) r_{m}\right)$. On the other hand, $D_{m}^{\prime}=\psi_{m}\left(B\left(2 \pi i t_{m}, 100\left|t_{m+1}\right|\right)\right.$ contains the disk $B\left(c_{m}, 100 \delta_{m}\left(\alpha\left(100 \delta_{m}\right)\right)^{-1} r_{m}\right)$. We conclude that if $\delta_{m}<x_{0}$ where $x_{0}>0$ is the solution of equation

$$
100\left(1-100 x_{0}\right)^{2}=17\left(1-x_{0}\right)^{-2}
$$

then

$$
B\left(c_{m+1}\right) \subset B\left(c_{m}, 17 \delta_{m} \alpha\left(\delta_{m}\right) r_{m}\right) \subset B\left(c_{m}, 100 \delta_{m}\left(\alpha\left(100 \delta_{m}\right)\right)^{-1} r_{m}\right) \subset D_{m}^{\prime} .
$$

The condition $\delta_{m}<x_{0}$ means that $2\left|t_{m+1}\right|<d_{m} x_{0}$. This holds if $(9 / 10)^{q_{m}}<$ $\left(d_{m} / 2\right) x_{0}$ which is aparently always the case if $q_{m}$ is big enough. Thus if $n$ is big, the conclusion (ii) holds. Lemma is proved.

The lemma implies that for all $m$,

$$
D_{m+1}^{\prime} \subset B_{m+1} \subset B\left(c_{m+1}\right) \subset D_{m}^{\prime} .
$$

Since the limb $L\left(W^{m}, t_{m}\right)$ is contained in $B\left(c_{m}\right)$ and the diameters of $D_{m}^{\prime}$ is by a definite factor smaller than the diameter of $B\left(c_{m}\right)$, we conclude that the limbs $L\left(W^{m}, t_{m}\right)$ shrink to a point $c_{*}$. This proves the conclusion (1) of the Theorem. Moreover, we have:

$$
\left\{c_{*}\right\}=\cap_{m=0}^{\infty} B_{m}=\cap_{m=0}^{\infty} D_{m}^{\prime} .
$$

Based on this and on Lemma 6.1, it is not difficult to prove the conclusion (2).

Indeed, let us denote by $O_{m}(c)$ the $n_{m}$-periodic orbit of $f_{c}$, which is attracting if $c \in W^{m}, m=0,1,2, \ldots$. As we know, $O_{m}$ extends holomorphically for $c$ in the wake $\left(W^{m}\right)^{*}$ of $W^{m}$. Moreover, Lemma 6.3 tells us that for each $m=0,1,2, \ldots$, the orbit $O_{m+1}$ extends holomorphically to the disk $B_{m}$ near the root $c_{m}$ of the wake $\left(W^{m+1}\right)^{*}$.

Since $c_{*} \subset D_{m}^{\prime} \subset B_{m}$ and $c_{m+1} \subset D_{m}$, Corollary 6.1 allows us to write:

$$
d\left(O_{m}\left(c_{*}\right), O_{m}\left(c_{m}\right)\right)<6\left(\frac{10(100)^{1 / q_{m}} \epsilon_{m}}{9}\right)^{q_{m}}<6(2 \Delta)^{q_{m}}<\Delta
$$

and

$$
d\left(O_{m+1}\left(c_{m+1}\right), O_{m}\left(c_{m}\right)\right)<7 \epsilon_{m} .
$$

By the triangle inequality for $d$, we get from ( 34):

$$
d\left(O_{m+1}\left(c_{m+1}\right), O_{0}\left(c_{0}\right)\right)<7 \sum_{k=0}^{m} \epsilon_{k}
$$


and then (33) implies

$$
d\left(O_{m+1}\left(c_{*}\right), O_{0}\left(c_{0}\right)\right)<7 \sum_{k=0}^{m} \epsilon_{k}+\Delta<8 \Delta .
$$

On the other hand, since $O_{0}\left(c_{0}\right)$ is a neutral $n$-periodic orbit of $f_{c_{0}}$, the distance of $O_{0}\left(c_{0}\right)$ to zero is bigger than $4^{-n}$. Hence, we have if $\Delta=4^{-n} / 16$, then, for all $m=0,1,2, \ldots$, every point of $O_{m}\left(c_{*}\right)$ is away from the origin by the distance at least $4^{-n} / 2$.

It is known that this implies the non local connectivity of $J_{c_{*}}$ (see [20] for a detailed proof, although our description is closer to [16]). Let us outline a proof. Denote by $\tau_{m}, \tau_{m}^{\prime}$ two external arguments in the $c$-plane to the root $c_{m}$ of the wake of $W^{m}$. By the formula ( [27), $\left|\tau_{m+1}-\tau_{m+1}^{\prime}\right| \leq\left(2^{n_{m}}\right)^{2} /\left(2^{n_{m} q_{m}}-1\right) \rightarrow 0$ as $m \rightarrow \infty$, that is $\tau_{m}, \tau_{m}^{\prime}$ tend to some $\tau^{*}$.

On the other hand, for every $c$ in the wake of $W^{m+1}$, we have the following picture in the dynamical plane of $f_{c}$ (see beginning of the present Section): two external rays of $f_{c}$ with arguments $\tau_{m+1}$ and $\tau_{m+1}^{\prime}$ land at a single point $b_{m}(c)$, which is a point of the repelling periodic orbit $O_{m}(c)$. Moreover, $c$ lies in the sector bounded by these two external rays and disjoint with the origin.

Now assume that the Julia set of $f_{c_{*}}$ is locally connected. Then this discussion implies that, in the dynamical plane of $f_{c_{*}}$, the external argument of $c_{*}$ is equal to $\tau_{*}$ and since $\tau_{m} \rightarrow \tau_{*}$, also $b_{m}\left(c_{*}\right) \rightarrow c_{*}$. Taking a preimage by $f_{c_{*}}$, it gives us a sequence of points of the sets $O_{m}\left(c_{*}\right)$ which tends to the origin, a contradiction with the fact that the orbits $O_{m}\left(c_{*}\right)$ stay away from the origin.

\subsection{Proof of Theorem 2}

Here we prove a more general Theorem 7 . Theorem 2 announced in the Introduction is an immediate corollary of this together with Definition 5.1 and sufficient conditions (21) $-($ 22) .

Theorem 7 Let $n \geq 1$. Let

$$
t_{0}, t_{1}, \ldots, t_{m}, \ldots
$$

be any sequence of rational numbers $t_{m}=p_{m} / q_{m} \in(-1 / 2,1 / 2]$ which satisfy the following properties: if we denote $n_{0}=n, n_{m}=n q_{0} \ldots q_{m-1}, m>0$, then, for all $m$ large enough, $t_{m}$ is $n_{m}$-deep, and also

$$
\sum_{m=1}^{\infty}\left|t_{m}\right|^{1 / q_{m-1}}<\infty
$$

Given a hyperbolic component $W$ of the Mandelbrot set of period $n$, consider a sequence of hyperbolic components $W^{m}: W_{0}=W$, and, for $m>0, W^{m}=$ 
$W^{m-1}\left(t_{m-1}\right)$, i.e. $W^{m}$ touches the hyperbolic component $W^{m-1}$ at a point with the internal argument $t_{m-1}$. For every $m$, consider the $t_{m}$-limb $L\left(W^{m}, t_{m}\right)$ of $W^{m}$ (it contains $\left.W^{m+1}\right)$. Then the sequence of limbs $L\left(W^{m}, t_{m}\right)$ shrink to a unique point $c_{*}$, the Mandelbrot set is locally connected at $c_{*}$, and the map $f_{c_{*}}$ is infinitely renormalizable with non locally connected Julia set.

Let $m_{0}$ be large enough, so that $\left|t_{m+1}\right|<(9 / 10)^{q_{m}}$ and $t_{m}$ is $n_{m}$-deep for every $m \geq m_{0}$. Then the sequence $t_{m_{0}}, \ldots, t_{m}, \ldots$ satisfies the conditon (a) of Theorem 6 with $n=n_{m_{0}}$. Hence, by the conclusion (1) of Theorem [6 the limbs $L\left(W^{m}, t_{m}\right)$ shrink to a unique point $c_{*}$.

It remains to show that the Julia set $J_{c_{*}}$ of $f_{c_{*}}$ is not locally connected. We show that whether $J_{c_{*}}$ is locally connected or not depends only on a tail of the sequence $t_{0}, \ldots, t_{m}, \ldots$. Let us introduce the following notation. Let $W_{0}$ be the 1 hyperbolic component (the main cardioid). Given $k$, let's start with the component $W_{0}$ and the tail $T_{k}=\left\{t_{k}, t_{k+1}, \ldots\right\}$ in place of $W$ and $\left\{t_{0}, t_{1}, \ldots\right\}$. Then we get a sequence of hyperbolic components $W_{0}^{k, m}, m \geq k$, where $W_{0}^{k, k}=W_{0}$ and, for $m>k$, the component $W_{0}^{k, m}$ touches $W_{0}^{k, m-1}$ at the point with internal argument $t_{m-1}$. We have proved that, for every $k \geq m_{0}$, the sequence of $\operatorname{limbs} L\left(W_{0}^{k, m}\right)$, $m=k, k+1, \ldots$ shrinks to a unique point $c_{*}^{k}$. Now, we have

Proposition 4 For every $k$ large enough, the Julia set of $f_{c_{*}^{k}}$ is not locally connected.

Proof. If $c$ is in the boundary of the main cardioid, the neutral fixed point $\alpha(c)$ of $f_{c}$ has modulus $1 / 2$. Since $t_{k} \rightarrow 0$, by continuity (and Yoccoz's bound), there is $\delta_{0}>0$, such that, for every $k$ large enough, for $c$ in the boundary of $W\left(t_{k}\right)$, the neutral periodic orbit of $f_{c}$ (of period $q_{k}$ ) stays away from 0 by at least $\delta_{0}$. If we increase $k$ further, one can assume that

$$
\sum_{m=k}^{\infty}\left|t_{m+1}\right|^{1 / q_{m}}<4^{-q_{k}} / 16
$$

and also $4^{-q_{k}} / 2<\delta_{0}$. Define $\delta=\delta_{0}-4^{-q_{k}} / 2$. Now we can apply Theorem 6 to $q_{k}$ instead of $n$ and to the sequence $t_{k+1}, t_{k+2}, \ldots$ taking into account ( 35). It shows that all corresponding periodic orbits at the parameter $c_{*}^{k}$ will stay away from the origin by at least $\delta$.

Let us come back to the map $f_{c_{*}}$. Next statement will finish the proof that $J_{c_{*}}$ is not locally connected.

Proposition 5 For every $k$, there is a restriction $f_{c_{*}}^{n_{k}}: U_{k} \rightarrow V_{k}$, such that this is a polynomial-like map of degree 2 , which is quasi-conformally conjugate to $f_{c_{*}^{k}}$. 
Proof. Consider first the wake of $W^{k-1}$. For $c$ in this wake, $f_{c}$ has a periodic orbit $O_{k-1}(c)$ of period $n_{k-1}$ which is attracting iff $c \in W^{k-1}$ and is holomorphic in $c$. Now consider the wake $\left(W^{k}\right)^{*}$ of the next component $W^{k}$. Denote also $a_{k}$ the root of $W^{k}$. If $c=a_{k}$, then $O_{k-1}\left(a_{k}\right)$ is neutral with the multiplier $\exp \left(2 \pi i t_{k-1}\right)$ : there are $q_{k-1}$ petals attached to each point of $O_{k-1}\left(a_{k}\right)$, and $f_{a_{k}}^{n_{k-1}}$ acts (locally) on them as a rotation with the totation number $t_{k-1}$. If $c \in\left(W^{k}\right)^{*}$, then $O_{k-1}(c)$ is repelling, and has the rotation number $t_{k-1}$. There exists a point $b_{k-1}(c)$ of $O_{k-1}(c)$, such that for $c \in\left(W^{k}\right)^{*} \cup\left\{a_{k}\right\}$, there are two external rays $R_{k}(c), \tilde{R}_{k}(c)$ of arguments $\tau\left(a_{k}\right), \tilde{\tau}\left(a_{k}\right)$ landing at $b_{k-1}(c)$, such that the component of the plane bounded by these two rays that contains $c$ contains no other ray to the orbit. There are two topological disks $c \in S_{k}^{\prime}(c) \subset S_{k}(c)$, such that, $S_{k}(c)$ is bounded by $b_{k-1}(c)$, the rays $R_{k}(c), \tilde{R}_{k}(c)$ and by some equipotential, and $f_{c}^{n_{k}}: S_{k}^{\prime}(c) \rightarrow S_{k}(c)$ is a proper map of degree 2. By a "thickening" of $S_{k}(c)$, one can turn it into a polynomial-like map $P_{k, c}: U_{k, c} \rightarrow V_{k, c}$, see [17 for details.

If $c \in B:=\left\{c_{*}\right\} \cup \cup_{m=k}^{\infty}\left(W^{m} \cup\left\{a_{k}\right\}\right)$, we claim that the Julia set of $P_{k, c}$ : $U_{k, c} \rightarrow V_{k, c}$ is connected. Indeed, consider the iterates $f_{c}^{n_{k} j}(c), j=0,1, \ldots$. If $c$ is close to $a_{k}$ and in $W^{k}$, then they converge to $b_{k}(c)$. On the otehr hand, neither of these iterates cannot cross the boundary of $S_{k}^{\prime}(c)$ when $c \in B$, because $f_{c}$ is not Misiurewich map for such $c$. This proves the claim.

By the Straightening Theorem [5], for every $c \in B$, there is a unique $\nu(c) \in M$, such that $P_{k, c}: U_{k, c} \rightarrow V_{k, c}$ is hybrid equivalent to $f_{\nu(c)}$. Moreover, by [5], the map $c \mapsto \nu(c)$ is continuous (it follows essentially from the compactness of $K$ quasiconformal maps and Proposition 7 of [5]). We need to show that $\nu\left(c_{*}\right)=c_{*}^{k}$. By continuity, it is enough to show that, for every root $a_{m}$ with $m>k, \nu\left(a_{m}\right)$ is the root $a_{m}^{k}$ of the hyperbolic component $W_{0}^{k, m}$. Let us prove it by induction in $m$.

(1) $m=k+1$. Notice that if $c$ is close to $a_{k}$ and in $W^{k}$, then $P_{k, c}: U_{k, c} \rightarrow V_{k, c}$ has an attracting fixed point: the point $b_{k}(c)$ of $O_{k}(c)$ which lies in $S_{k}^{\prime}(c)$ and coincides with $b_{k-1}(c)$ when $c=a_{k}$. This attracting fixed point persists as $c \in W^{k}$ (periodic points cannot leave $S_{k}^{\prime}(c)$ ), and when $c=a_{k+1}$, it has the multiplier $\exp \left(2 \pi i t_{k}\right)$. Hence, the conjugate map $f_{\nu\left(a_{k+1}\right)}$ has a neutral fixed point, and it interchanges the petals at this point with the same rotation number $t_{k}$. It follows, $\nu\left(a_{k+1}\right)=a_{k+1}^{k}$, the unique point in the boundary of the main cardioid with the internal argument $t_{k}$.

(2) assume that $\nu\left(a_{m}\right)=a_{m}^{k}$ holds for some $m \geq k+1$. Then a similar argument shows that $\nu\left(a_{m+1}\right)=a_{m+1}^{k}$. 


\subsection{Maps with unbounded combinatorics}

Let us consider the case $n=1$, that is we start with the main cardioid. Then we can reformulate Theorem 2 as follows.

Theorem 8 Let a sequence $p_{m} / q_{m}, m=0,1, \ldots$, of non-zero rational numbers in $(-1 / 2,1 / 2)$ be such that:

$$
\begin{gathered}
\sum_{m=1}^{\infty}\left|\frac{p_{m}}{q_{m}}\right|^{1 / q_{m-1}}<\infty, \\
p_{m}>\left(2 B_{0}\right) 4^{n_{m}}, \quad 2 n_{m}^{2}<\frac{p_{m}^{2}}{q_{m}},
\end{gathered}
$$

for all $m$ large enough, where $n_{m}=q_{0} \ldots q_{m-1}$.

Let $f_{c}$ be an infinitely renormalizable polynomial with the following combinatorial data.

(1) The renormalization periods consists of the sequence $\left\{n_{m}\right\}_{m=1}^{\infty}$.

(2) Denote $J_{0}=J\left(f_{c}\right)$, and, for every $m>0, J_{m}$ be the Julia set of the renormalization of period $n_{m}$, which contains 0 . Let $\alpha_{m}$ and $\beta_{m}$ be the $\alpha$ (i.e. separating) and $\beta$ (i.e. non separating) fixed points of $f_{c}^{n_{m}}: J_{m} \rightarrow J_{m}$. We assume that, for every $m, \beta_{m+1}=\alpha_{m}$, and the rotation number of $\alpha_{m}$ w.r.t. the map $f_{c}^{n_{m}}: J_{m} \rightarrow J_{m}$ is equal to $p_{m} / q_{m}$.

Then there exists a unique polynomial which satisfies the conditions (1)-(2), and its Julia set is not locally connected.

\section{References}

[1] Carleson, L. \& Gamelin, T.: Complex dynamics. Springer-Verlag, 1993

[2] Cheritat, A.: Estimates on the speed of explosion of the parabolic fixed points of quadratic polynomials and applications. Preprint Nov. 23, 1999.

[3] Douady, A. \& Hubbard, J.H.: Iteration des polynomes quadratiques complexes. C.R.A.S., t. 294 (1982), 123-126

[4] Douady, A. \& Hubbard, J.H.: Etude dynamique des polynomes complexes. Pub. Math. d'Orsay, 84-02 (1984), 84-04 (1985)

[5] Douady, A. \& Hubbard, J.H.: On the dynamics of polynomial-like maps. Ann. Sc. Ec. Norm. Sup., v. 18 (1985), 287-343

[6] Eremenko, A. \& Levin, G.: Estimating the characteristic exponents of polynomials (in Russian). Theor. Funk., Funkts. Anal. i Prilozhen, no. 58 (1992), 30-40 (1993). Translation in Journal of Math. Scienes, 85 (1997), no. 5, 21642171. 
[7] Eremenko, A. \& Levin, G.: Periodic points of polynomials (in Russian). Ukrain. math. Zh. 41 (1989), no.11, 167-1471. Translation in Ukrainian Math. J., no.11 (1990), 1258-1262

[8] Goluzin, G. M.: Geometric theory of functions of complex variable. Moscow, 1966.

[9] Guckenheimer, J. \& McGehee, R.: A proof of the Mandelbrot N-square conjecture. Institut Mittag-Leffler. Report No. 15, 1984. (See first author's web page.)

[10] Hubbard, J. H.:Local connectivity of Julia sets and bifurcation loci: three theorems of J.-C. Yoccoz. In: "Topological Methods in Modern Mathematics." Publish or perish, 1993

[11] Levin, G.: On explicit connections between dynamical and parameter spaces. Journal d'Analyse Mathematique, 91( 2003), 297-327

[12] Levin, G.: On bounds for multipliers of periodic points of holomorphic mappings (Russian). Sibirsk. Math. Zh. 31 (1990), no. 2, 104-110. Translation in Siberian Mathematical Journal, v.31, no.2 (1990), 273-278.

[13] Levin, G.: On Pommerenke's inequality for the eigenvalue of fixed points. Colloquium Mathematicum, LXII (1991), Fasc. 1, 168-177.

[14] Levin, G. \& Sodin, M.: Polynomials with disconnected Julia sets and Green maps. Hebrew Univ. of Jerusalem, Preprint 23/1990-91.

[15] Levin, G.: Disconnected Julia set and rotation sets. Ann. Scient. Ec. Norm. Sup., 4 series, t. 29 (1996), 1-22.

[16] Milnor, J.: Non locally connected Julia sets constructed by iterated tuning. Birthday lecture for "Douady 70". Revised May 26, 2006, SUNY, 28 pp. (See author's web page.)

[17] Milnor, J.: Periodic orbits, external ray and the Mandelbrot set: an expository account. "Geometrie Complexe et Systemes Dynamiques". Colloque en l'honneur d'Adrien Douady (Orsay, 1995). Asterisque 261 (2000), 277-331

[18] Petersen, C. L.: On the Pommerenke-Levin-Yoccoz inequality. Ergodic Theory Dynam. Systems 13 (1993), no 4, 785-806.

[19] Pommerenke, Ch.: On conformal mappings and iteration of rational functions. Complex Variables Th. and Appl. 5 (1986), no. 2-4

[20] Sorensen, D. E. K.: Infinitely renormalizable quadratic polynomials with nonlocally connected Julia set. J. Geom. Anal. 10 (2000), 169-206. 